\title{
A Multiobjective Evolutionary Approach to a Medium- Sized Multicriteria Ranking Problem
}

\author{
Juan Carlos Leyva López ${ }^{1}$, Jesús Jaime Solano Noriega² ${ }^{2}$ Diego Alonso Gastélum Chavira ${ }^{1}$, María de los \\ Dolores Sánchez Castañeda ${ }^{3}$ \\ ${ }^{1}$ Universidad de Occidente \\ ${ }^{2}$ Universidad Autónoma de Ciudad Juárez \\ ${ }^{3}$ Centro de Investigación en Matemáticas \\ juan.leyva@udo.mx, jaimesolanonoriega@hotmail.com, diego.gastelum@udo.mx, maria.sanchez@cimat.mx
}

\begin{abstract}
The framework of multiobjective optimization is used to tackle the multicriteria ranking problem. The conceptual advantages of the multiobjective formulation are discussed and a new multiobjective evolutionary algorithm is introduced with the purpose of transforming a known valued outranking relation into an antisymmetric crisp outranking relation, on a set of classes of alternatives, where the elements of each class are indifferent each other, and with this as a background, we propose a recommendation for ranking problems of medium-sized set of alternatives. The performance of the algorithm is evaluated on a test problem. It was capable of producing a high-quality recommendation.
\end{abstract}

Keywords: Multicriteria analysis; Valued outranking relations; Multiobjective evolutionary algorithms; Ranking procedure.

\section{Introduction}

A Multiple Criteria Decision Analysis provides two major approaches of constructing a global preference model from an actor involved in the decision process. The first one is the functional model, which has been widely used within the framework of multi-attribute utility theory (e.g. (Keeney and Raiffa, 1976; French, 1986; Triantaphyllou, 2000). The second one is the relational model, which has its most known representation in the form of a crisp or fuzzy outranking relation (e.g. (Roy, 1990)).

The multiple criteria aggregation methods allow us to construct a recommendation from a set of alternatives based on the preferences of a decision maker. In the functional approach, the recommendation is immediately deduced from the preferences aggregation process. When the aggregation model of preferences is based on the relational approach, a special treatment is required, but some non-rational violations of the explicit global model of preferences could happen.

This paper is concerned with the relational approach to Multiple Criteria Decision Aid (MCDA). Methods related to this approach, including the well-known family of ELECTRE methods, are often presented as the combina- tion of two phases: aggregation (or construction) and exploitation. For the multicriteria ranking problem, the second phase is usually carried out with a ranking method.

Most respected text on MCDA (Vincke, 1992; Doumpos and Zopounidis, 2002 ; Figueira et al., 2005; Roy, 2006; Ehrgott et al., 2010; etc.) define the ranking problematic _P.y informally, e.g. (Roy, 1996) defines the ranking problematic as:

Definition (Roy, 1996). The ranking problematic P. $\gamma$ presents the problem in terms of ranking the actions of $\mathrm{A}$, that is, of directing the investigation towards determining an order defined on a subset of A so as to be able to determine those actions that could be considered as "sufficiently satisfactory" based on a preference model, while keeping in mind that A might evolve. This problematic leads to a recommendation or simple participation that either: suggest a partial or complete order formed by the classes containing actions considered equivalents.

The use of informal definitions such as this reflects one of the prevailing and fundamental problems in MCDA: the difficulty of providing a single formal (but sufficiently broad) definition of the concept of a ranking. The concept of a ranking is a generalization of what decision makers perceive as a hierarchy of a set of alternatives in decreasing order of preferences, a decision maker intuition which is inherently difficult to capture by means of individual objective criteria. We illustrate this in Figure 1.
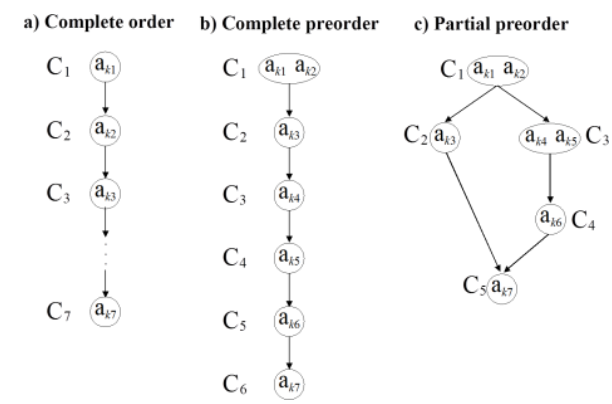

Fig. 1: Different ranking structures. Rankings may be a complete order, or a complete preorder, or a partial preorder. While each of these features may pose problems to specific ranking criteria, the main ranking structure can be perceived easily by decision makers. 
On the other hand, most existing ranking methods for exploiting a known valued outranking relation attempt, explicitly or otherwise, to optimize just one such criterion, and it is this confinement to a particular ranking property that explains the fundamental discrepancies observable between the recommendations produced by different algorithms on the same data, and will cause a ranking method to fail (as judged by means of external knowledge) in a context where the criterion employed is inappropriate. In practice, this problem can be alleviated through the application and comparison of multiple ranking methods (see e.g. Guitouni and Martel (1998)).

Initially, ranking algorithms were designed to capture the decision maker notion of a ranking, however, with the increased complexities of some kinds of decision problems, traditional ranking algorithms sometimes do not perform very well, especially in instances where there are a medium-sized set of alternatives and/or there are a lot of intransitivities or incomparabilities between pairs of alternatives. But with the emergence of new mathematical techniques and with the enormous computer performance that exist nowadays, it is possible design new ranking algorithms for the application to the ranking of complex data sets not interpretable by decision makers. Applying new rules, further than only the traditional solutions and ideas, this kind of procedures should exploit the aggregation model of preferences of the decision maker and be able to provide a recommendation in form of a ranking.

The aim of the proposed approach is twofold: first. We want to partition a medium-sized set of alternatives into $\mathrm{k}$ classes; second, based solely on the initially provided information, we want to elicit the antisymmetric crisp outranking relation between the determined classes. The particular advantage of the proposed approach is to integrate partition and relation between classes into the optimization process that the multiobjective evolutionary algorithm performs. The result of our method is thus given by a partition $P_{k}(A)$ of $A$ into a set of classes $C=\left\{C_{1}, C_{2}, \ldots, C_{k}\right\}$ and a kxk crisp antisymmetric outranking relation $S_{P_{k}(A)}$ on that partition.

In the next section, the ranking problematic is presented. The single-objective ranking is explained in Section 3. Section 4 presents the multiobjective ranking, and with these as the background, we present a multiobjective evolutionary algorithm to a medium-sized ranking problem in Section 5. A test problem and the computational results are given in Section 6, and finally, in Section 7, several conclusions are discussed.

\section{The Ranking Problematic P. $\gamma$}

The objective of ranking problematic is to aid the DM through a ranking that is obtained by placing all, or simply the "most attractive" actions into equivalence classes that are completely or partially ordered according to pref- erences. P. $\gamma$ results in a ranking or an ordering procedure.

The analyst could have considered a procedure to rank the actions according to decreasing preference. Roy said that it can be seen as a "competition" where we can think of comparing actions so that they can be grouped into classes that can be ordered. The classes would group actions that are considered equivalent, at least for those classes near the top of the order.

Roughly, one can imagine that the "true" preferences of a decision maker are represented by a crisp or valued outranking relation. A ranking, then, can be seen as "revealed" preferences.

In adopting this problematic, one tries to use available information as much as possible to compare the elements of $A$ among each other so as to determine classes for the elements in $A$ and a ranking of these classes. Such a ranking or ordering, which is developed to aid the decision maker, must reflect a certain degree of importance or priority that the decision maker gives to each element of $A$. The ranking is designed to help his/her think about the problem, to guide his/her discussions with other stakeholders and, more generally, to serve as a framework for approaching the next critical point of the decision process (Roy, 1996).

The ordering procedure considered in this problematic consist of assigning a "rank" to each action in $A$, where two actions are assigned the same rank whenever the data do not allow a distinction between them with respect to the next critical point of the analysis. The ranking of the classes allows an ordering, complete or partial, representative of preferences.

The classes of P. $\gamma$ are not determined from an a priori definition. Rather, the significance of a class is relative, in that it depends on its position in the overall ordering. One should not necessarily require that the classes of $P . \gamma$ form a complete order. The poor quality of the data, the conflicting criteria, and the multiple value systems involved might make it difficult or artificial to develop a complete order. In certain cases, a partial order, which does not necessarily position a class relative to every other class, can be quite useful. Moreover, the classes obtained in P. $\gamma$ are defined neither with the choice of a single best action in mind nor with the objective to be as small as possible (Roy, 1996).

\section{Single-objective ranking}

We have outlined above that the quality of a ranking is ultimately defined in terms of decision maker knowledge. However, ranking algorithms are unsupervised methods and external knowledge of any kind is not usually available during the ranking process. In order to assess the quality of individual rankings, ranking algorithms therefore rely on the adoption of internal criteria as proxies for the unknown "correct hierarchical classification." The re- 
sulting transformed ranking problem $(\Omega, P)$ can then be formally defined as an optimization problem: determine the ranking $C^{*}$ for which

$$
P\left(C^{*}\right)=\min _{C \in \Omega} P(C)
$$

Where $\Omega$ is the set of feasible rankings (here we take this to be any kind of ordering of the elements of $A$ ), $C$ is a ranking of a given set of alternatives $A$, and $P$ is the (internal) criterion function. $P$ is always based on the notion of preference between data items, which is provided as the input, i.e. only the relationships between individual data items are known. We assume $P$ is to be minimized without loss of generality. Examples of criterion functions are the score functions (Net Flow Rule), choice functions (Distillation method), distance-based functions (Kendall's distance), etc. Different formulations of the ranking vary in the optimization criterion $P$ used.

\section{Multiobjective ranking}

An alternative approach may be the consideration of ranking as a multiobjective optimization problem as suggested by Leyva and Araoz (2013). In a multiobjective ranking problem $\left(\Omega, P_{1}, P_{2}, \ldots, P_{m}\right)$ we aim to determine the ranking $C^{*}$ for which

$P_{t}\left(C^{*}\right)=\min _{C \in \Omega} P_{t}(C), \quad t=1,2, \ldots, m$

Where $\Omega$ is the set of feasible rankings (here we take this to be any kind of ordering of the elements of $A$ ), $\mathrm{C}$ is a ranking of a given set of data $A$, and $P_{t}: t=1,2, \ldots, m$ is a set of $m$ different (single) criterion function. Usually no single best solution for this optimization task exists, but, instead, the framework of Pareto optimality is embraced.

The set of Pareto-optimal solutions to a multiobjective ranking problem $\left(\Omega, P_{1}, P_{2}, \ldots, P_{m}\right)$ always comprises the optimal solutions to the single-objective ranking problems $\left(\Omega, P_{1}\right),\left(\Omega, P_{2}\right), \ldots,\left(\Omega, P_{m}\right)$ (Leyva and Araoz, 2013). For ideal single and multiobjective rankings algorithms (i.e. algorithms that always identify all globally optimal solutions, and the entire Pareto-optimal set, respectively), we therefore trivially know that the multiobjective algorithm will always find a solution as good o better (equal in terms of the ranking objective optimized and equal o possible better in terms of external knowledge) than those of the single-objective algorithms. In situations where the best solution corresponds to a trade-off between the different objectives only the multiobjective ranking algorithm will be able to find it.

An example of a set of alternatives, for which this is relevant, is shown in Figure 2. Here different possible ranking solutions are plotted in two objective space and it can be seen that the "correct solution" represent a trade-off between the two objectives.

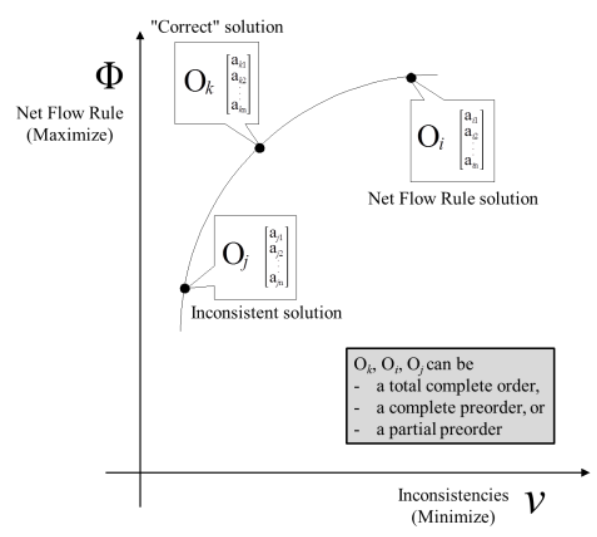

Fig. 2: Different possible ranking solutions.

\section{A Multiobjective Evolutionary Algorithm to a Me- dium-Sized Ranking Problem}

The aim of this paper is to present a method dedicated to multicriteria ranking problem with a medium-sized set of alternatives. It is worth noting that the term multicriteria ranking can be understood in the sense of determining a hierarchical partition that simultaneously optimizes several objective functions. In such cases, ranking can be seen as a special case of multiobjective optimization and well performing approaches such as evolutionary multiobjective optimization (EMO) algorithms could be successfully applied. Several methods have been proposed in the literature for tackling the multicriteria ranking as a multiobjective optimization problem, where we are looking for a total order between the alternatives. For additional details see for instance (Leyva and Araoz, 2013). In this paper we follow this interpretation in a slightly modified form. We will use the term multicriteria ranking to point out ranking methods based on asymmetric relations resulting from the application of a given multicriteria procedure in the sense of determining a hierarchical partition (an antisymmetric relation on the classes) that simultaneously optimizes several objectives.

In this case, multicriteria ranking can be seen as a special case of multiobjective optimization problem and we proposed in this paper an evolutionary multiobjective optimization algorithm for tackling this type of multicriteria ranking problem. To the best of our knowledge, this approach has not previously been proposed.

\subsection{Modeling the problem of ranking in MCDA}

In this section we present a way of modeling the different objectives of ranking in MCDA with a medium-sized set of alternatives. We first present a general way in which alternatives and classes of alternatives can be compared to each other, and then, using this we highlight and model the ranking objectives. 


\subsubsection{Comparing alternatives and set of alternatives}

Let $A$ denote a set of decision alternatives and $S_{A}^{\sigma}$ a valued outranking relation. Each potential solution in the population is associated with a number $\lambda$, the cut level, where $0 \leq \lambda \leq 1$. For each value of the cut level $\lambda$, we can induce a crisp outranking relation $S_{A}^{\lambda}$. In $S_{A}^{\lambda}$ we can deduce the follows preference relations:

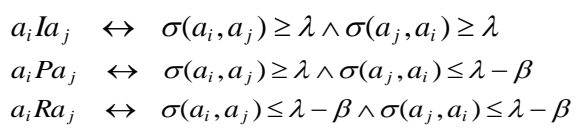

\subsection{The multiobjective evolutionary algorithm}

In this subsection, we present a multiobjective evolutionary algorithm based on a posterior articulation of preferences, which is able to exploit a known valued outranking relation with the purpose of constructing a recommendation for the multicriteria ranking problem with a mediumsized set of alternatives. The algorithm borrows fundamental elements from MOGA (Fonseca and Fleming, 1993). In the following subsections, we present in further detail the fundamental aspects of the algorithm.

5.2.1- Representation of a potential solution in the ranking problem

We use a locus-based adjacency representation (Park and Song, 1998) as shown in Figure 3. In this graph-based representation, each individual $p$ consists of $m$ genes $p_{1}, p_{2}, \ldots, p_{m}$ and each gene $p_{i}$ can take allele values $j$ between 1 and $m$. a value of $j$ assigned to the gene $p_{i}$ is interpreted as a link from alternative $i$ to $j$, in the resulting class solution they will be in the same class. The decoding of this representation requires the identification of all connected component. All alternatives belonging to the same connected component are then assigned to one cluster. The decoding step takes linear time (Cormen et al., 2001).

\section{a) Locus-based adjacency representation}

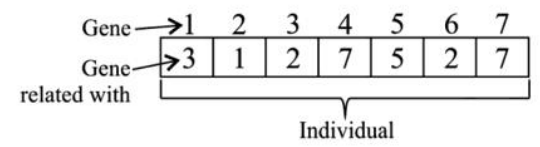

b) Graph representation

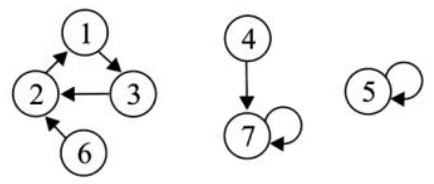

c) Clustering representation

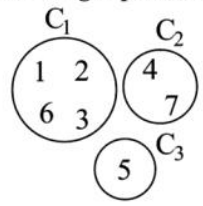

Fig. 3: Illustration of the locus-based adjacency representation. A set of 7 alternatives $A=\left\{a_{1}, a_{2}, \ldots, a_{7}\right\}$ is partitioned. Figure (a) shows one possible genotypes of an individual of the population. It is transformed into the graph structure shown in Figure

(b). Every connected component with this graph is interpreted as an individual class, as visualized by the circles in Figure (c).

The locus-based adjacency encoding scheme has several major advantages; most important, there is no need to fix the number of classes in advance, as it is automatically determined in the decoding step. Hence, it is possible to evolve and compare solutions with different number of classes in just one run of the evolutionary algorithm.

\subsubsection{Objective functions}

\subsubsection{Maximizing the cutting level $\lambda$}

From the valued outranking relation $S_{A}^{\sigma}$, it is possible to define a family of nested crisp outranking relations $S_{A}^{\lambda}$ $\left(S_{A}^{\lambda}=\{(a, b) \in A x A: S(a, b) \geq \lambda\}, \quad \lambda \in\left[\lambda_{0}, 1\right]\right)$; these crisp relations correspond to $\lambda$-cuts of $S_{A}^{\sigma}$, where the cutting level $\lambda$ represents the minimum value for $S_{A}^{\sigma}$ so that $a S_{A}^{\lambda} b$ is true (see (Fodor and Roubens (1994) for more details).

Each ranking is associated with a $\lambda$-cut, which is connected with the credibility level of a crisp outranking relation $S_{A}^{\lambda}$ defined on the set of alternatives. We want rankings for which the credibility level $\lambda$ is near 1 . This indicates to us that the ordering represented by the ranking with credibility level $\lambda$ is more trustworthy. We call this objective the maximum cut level objective. In practice, we use an additional condition for the credibility level $\lambda$. It is the function $f$ that does not permit $\lambda$ values to approach one because in this case, we could have many incomparable genes. The function $f$ is defined as follows: 
$f(\widetilde{p})=\left\{\begin{array}{l}\left(a_{k_{i}}, a_{k_{j}}\right): a_{k_{i}} \sim S a_{k_{j}} \text { and } a_{k_{j}} \sim S a_{k_{i}} \\ i=1,2, \ldots, m-1, j=2,3, \ldots, m, i<j\end{array}\right\} \mid, \quad f(\widetilde{p})$ is the number of incomparabilities between pairs of actions $\left(a_{k_{i}}, a_{k_{j}}\right)$ in the individual $\tilde{p}=a_{k_{1}} a_{k_{2}} \ldots a_{k_{m}}$ in the sense of the crisp relation $S_{A}^{\lambda}$. Note that the quality of a solution increases with decreasing $f$ score. In this case, we are interested in individuals whose $f$ values are equal (or close) to zero. This condition improves the comparability of $S$ on $A$.

\subsubsection{The min cut objective}

The min cut shown in equation 3 , aims to maximize the indifference with each class (see Figure 4). We proceed penalizing the pair of alternatives inside the class which are not indifferent. In the perfect case where all pairs of alternatives are indifferent each other then, there will not be penalizations.

$$
\operatorname{MinCut}=\sum_{m=1}^{k} A \operatorname{ssoc}\left(C_{m}\right)
$$

where

$$
\operatorname{assoc}(G)=\sum_{i \in G} \sum_{j \in G} \eta_{i j}
$$

In equation $3, \mathrm{k}$ is the number of classes, and $\eta_{i j}$ is the Boolean characteristic function $\eta$ which is defined as follows:

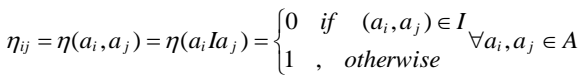

This quality is minimized in the corresponding multiobjective optimization problem. We call this objective the min cut objective.

\begin{tabular}{|c|ccc|ccc|ccc|}
\hline$K$ & $\mathrm{a}_{1}$ & $\mathrm{a}_{3}$ & $\mathrm{a}_{6}$ & $\mathrm{a}_{4}$ & $\mathrm{a}_{7}$ & $\mathrm{a}_{5}$ & $\mathrm{a}_{0}$ & $\mathrm{a}_{2}$ & $\mathrm{a}_{8}$ \\
\hline $\mathrm{a}_{1}$ & & $I$ & $I$ & $P$ & $P$ & $P$ & $P$ & $P$ & $P$ \\
$\mathrm{a}_{3}$ & & & $I$ & $P$ & $P$ & $P$ & $P$ & $P$ & $P$ \\
$\mathrm{a}_{6}$ & & & & $P$ & $P$ & $P$ & $P$ & $P$ & $P$ \\
\hline $\mathrm{a}_{4}$ & & & & & $I$ & $I$ & $P$ & $P$ & $P$ \\
$\mathrm{a}_{7}$ & & & & & & $I$ & $P$ & $P$ & $P$ \\
$\mathrm{a}_{5}$ & & & & & & & $P$ & $P$ & $P$ \\
\hline $\mathrm{a}_{0}$ & & & & & & & $I$ & $I$ \\
$\mathrm{a}_{2}$ & & & & & & & & & $I$ \\
$\mathrm{a}_{8}$ & & & & & & & & & \\
\hline
\end{tabular}

Fig. 4. Perfect case of ranking classes of alternatives

\subsubsection{The minimum pair-wise preference disagreement objective}

The crisp outranking relation of the alternatives $S_{A}^{\lambda}$ induces a crisp outranking relation $S^{*}$ between the determined classes in the following form:
For each pair of classes $\left(C_{r}, C_{q}\right), \quad r, q=1,2, \ldots, k$ we compute

$l_{r q}^{*}=\left\{\begin{array}{l}\underset{l_{r q} \in\{1,2,3,4\}}{\arg \max }\left(\sum_{a_{i} \in C_{r} a_{j} \in C_{q}} \sum_{l_{r q}}\left(a_{i}, a_{j}\right) \quad \text { if } \quad r \neq q\right. \\ 1 \quad \text { if } \quad r=q\end{array}\right.$

where

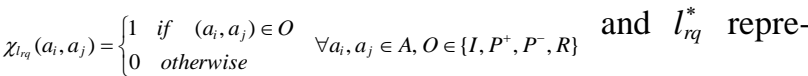
sent the crisp preference relation between the classes $C_{r}$ and $C_{q}$, De Smet and Eppe (2009) proof that this procedure leads to the optimal crisp outranking relation $S^{*}$.

Suppose that $C_{i} O C_{j}$, where $O \in\left\{I, P^{+}, P^{-}, R\right\}$. Suppose that $C_{i} P^{+} C_{j}$, is natural that in the beginning of the procedure, some pair of alternatives $\left(a_{r}, a_{s}\right), a_{r} \in C_{i}, a_{s} \in C_{j}$ does not be in concordance with $\left(C_{i}, C_{j}\right)$, i.e. [while $C_{i} P^{+} C_{j}$ in $S^{*}$ ], [ $a_{r} I a_{s}$, or $a_{r} P^{-} a_{s}$, or $a_{r} R a_{s}$ in $S_{A}^{\lambda}$ ]. In these conditions, we have an inconsistency between the aggregation model of preferences $S_{A}^{\lambda}$ and the crisp outranking relation of classes (See Figure 5).

\begin{tabular}{|c|ccc|ccc|ccc|}
\hline$K$ & $\mathrm{a}_{1}$ & $\mathrm{a}_{3}$ & $\mathrm{a}_{6}$ & $\mathrm{a}_{4}$ & $\mathrm{a}_{7}$ & $\mathrm{a}_{5}$ & $\mathrm{a}_{0}$ & $\mathrm{a}_{2}$ & $\mathrm{a}_{8}$ \\
\hline $\mathrm{a}_{1}$ & & $I$ & $I$ & $I$ & $P$ & $P$ & $P$ & $P$ & $P$ \\
$\mathrm{a}_{3}$ & & & $I$ & $P$ & $P$ & $P$ & $P$ & $P$ & $P$ \\
$\mathrm{a}_{6}$ & & & & $P$ & $P$ & $I$ & $P$ & $P$ & $P$ \\
\hline $\mathrm{a}_{4}$ & & & & $I$ & $I$ & $P$ & $I$ & $I$ \\
$\mathrm{a}_{7}$ & & & & & & $I$ & $P$ & $P$ & $I$ \\
$\mathrm{a}_{5}$ & & & & & & & $P$ & $P$ & $P$ \\
\hline $\mathrm{a}_{0}$ & & & & & & & & $I$ & $P$ \\
$\mathrm{a}_{2}$ & & & & & & & & & $I$ \\
$\mathrm{a}_{8}$ & & & & & & & & & \\
\hline
\end{tabular}

Fig. 5. Recommendation with inconsistencies between the aggregation model of preferences $S_{A}^{\lambda}$ and the crisp outranking relation of classes

We think that the quality of the final crisp outranking relation $S^{*}$ should also be judged according to the number of its discrepancies and concordances with $S_{A}^{\sigma}$ and the crisp outranking relations $S_{A}^{\lambda}$. Let $V$ be the set of strong discrepancies defined as:

$$
\begin{aligned}
& V=\left\{\left(a_{r}, a_{s}\right) \in A x A: a_{r} \in C_{i}, a_{s} \in C_{j}, a_{r} O_{1} a_{s}, C_{i} O_{2} C_{j},\right. \\
& O_{1} \in\left\{I, P^{+}, P^{-}, R\right\}, O_{2} \in\left\{I_{C}, P_{C}^{+}, P_{C}^{-}, R_{C}\right\}, O_{1} \neq O_{2}, \\
& i, j=1,2, \ldots, k\}
\end{aligned}
$$

Note that $O_{1} \neq O_{2}$ means that there is not concordance between the preference relations $a_{r} O_{1} a_{s}$ in $S_{A}^{\lambda}$ and 
$C_{i} O_{2} C_{j}$ in $S^{*}$. Also, $n_{V}$ is a function of the cut level $\lambda$, which counts the number of the pair-wise preference disagreements.

We quantify the number of preferences between alternatives into the crisp outranking relation $S_{A}^{\lambda}$ that are in disagreement in the sense of $S^{*}$. For the purpose of formulating the multiobjective optimization problem, we call this objective the minimum pair-wise preference disagreement objective.

Then, we use an evolutionary search to solve the multiobjective optimization problem:

$\operatorname{Min}(\operatorname{Min} \operatorname{Cut}(\widetilde{p})), \operatorname{Min}\left(n_{V}(\widetilde{p})\right), \quad \operatorname{Max}(\lambda(\widetilde{p}))$

Subject to:

$\widetilde{p}=\left[a_{k_{1}}, a_{k_{2}}, \ldots, a_{k_{m}}\right] \in \Omega$

$\lambda \in[0,1], \quad \lambda \geq \lambda_{0}, \lambda$

(where $\lambda_{0}$ is a minimumlevel of credibility)

where $\Omega$ is the set of feasible rankings (here we take this to be any kind of ordering of the elements of $A$ ), $\widetilde{p}$ is a ranking of a given set of data $A$. Usually no single best solution for this optimization task exists, but, instead, the framework of Pareto optimality is embraced.

\subsubsection{Fitness assignment procedure}

Most of the approaches of multiobjective decisionmaking seek the entire Pareto optimal set $P^{*}$, which in the jargon of Multiobjective Evolutionary Algorithms (MOEAs) is often denoted as $P_{\text {true }}$ (Coello et al. 2002). During the MOEA's execution, a "current" set of Pareto optimal solutions is determined at each EA generation and is termed $P_{\text {current }}(t)$, where $t$ represents the generation number. Many MOEA implementations also use a secondary population, storing non-dominated solutions found through the generations. This population is named $P_{\text {known }}(t)$. This term is also annotated by $\mathrm{t}$ to reflect its possible changes in membership during the execution of the MOEA. $P_{\text {known }}(0)$ is defined as the empty set $(\phi)$, and $P_{\text {known }}$ is defined as only the final set of solutions returned by the MOEA at termination. $P_{\text {current }}(t), P_{\text {known }}$, and $P_{\text {true }}$ are sets of MOEA genotypes; each set's corresponding phenotypes form an approximated Pareto front. The associated approximated Pareto front for each of these solution sets is called $P F_{\text {current }}(t), P F_{\text {known }}$, and $P F_{\text {true }}$.

Most of the methods based on MOEAs attempt to evolve a population toward the true Pareto frontier $P F_{\text {true }}$ The aim is that by the end of the run, $P_{\text {current }}=P_{\text {true }}$, $P_{\text {current }} \subset P_{\text {true }}, \quad$ or $\left\{\begin{array}{l}\vec{u}_{i} \in P F_{\text {current }}, \vec{u}_{j} \in P F_{\text {true }} \\ : \forall i, \forall j \quad \min \left[\text { distance }\left(\vec{u}_{i}, \vec{u}_{j}\right)\right]<\varepsilon\end{array}\right\}$, where the distance is defined over some norm.
To solve the multicriteria ranking problem using a MOEA, it is not necessary to seek the entire Pareto optimal set $P_{\text {true }}$ or the associated Pareto front $P F_{\text {true }}$ because of the fact that many of the non-dominated solutions are not of interest to the decision-maker. We will use the strategy of attempting to find in each EA generation the most promising and attractive solutions for the decision maker, which in our case are those individuals $\widetilde{p}(u, \Phi)$ whose $u, \Phi$ scores are close to a value of zero and have a sufficiently high value of $\lambda$. It is sufficient to seek a $r e$ stricted Pareto optimal set, which for our purpose is defined as follows:

$$
P_{\text {true }}^{\text {restricted }}=\left\{\begin{array}{l}
\tilde{p} \in P_{\text {true }}:\|(u(\tilde{p}), \Phi(\tilde{p}))\|_{\infty} \leq \varepsilon, \\
\text { where } \varepsilon \text { is a small } \\
\text { non-negative number, } \quad \lambda>0.5
\end{array}\right\}
$$

Based on this strategy, the proposed method attempts to evolve a population towards the true restricted Pareto frontier $\left(P F_{\text {true }}^{\text {restricted }}\right)$ by means of a succession of the restricted non-dominated solutions subset $P F_{\text {current }}^{\text {resticted }}(t)=\left\{P_{1}(t), P_{2}(t), \ldots, P_{n}(t)\right\}$. At each generation the method computes the non-dominated solutions for the ranking problem that is closest to the fixed aspiration level $(u, \Phi)$ with $u(\widetilde{p})=0$ and $\Phi(\widetilde{p})=0$ according to the Tchebycheff metric.

Fitness Assignment Procedure. The main steps are as follows:

Step 1 . Let $N$ be the population size. Choose a $\sigma_{\text {share }}$ (a dynamically updated procedure for fixing $\sigma_{\text {share }}$ is described later in Step 5). Initialise $\lambda_{j}=c_{j}\left(c_{j}\right.$ randomly chosen between 0 and 1$)$, and $\mu(j)=0$ for all possible ranks $j=1,2, \ldots, N$. Set the solution counter $i=1$.

Step 2. Calculate the number of solutions $\left(n_{i}\right)$ that dominate solution $i$. Compute the rank of the $i$-th solution as $r_{i}=1+n_{i}$. Increment the count for the number of solutions in rank $r_{i}$ by one, that is, $\mu\left(r_{i}\right)=\mu\left(r_{i}\right)+1$.

Step 3. If $i<N$, increment $i$ by one and go to step 2. Otherwise, go to step 4.

Step 4. Identify the maximum rank $r^{*}$ by checking the largest $r_{i}$ such that $\mu\left(r_{i}\right)>0$. Sorting according to rank and fitness averaging yields the following assignment of the average fitness $F_{i}$ to any solution $i=1,2, \ldots N$ :

$F_{i}=N-\sum_{k=1}^{r_{i}-1} \mu(k)-0.5\left(\mu\left(r_{i}\right)-1\right)$

For each solution $i$ with rank $r_{i}=1$, the above equation assigns a fitness equal to

$$
F_{i}=N-0.5(\mu(1)-1)
$$


which is the average value of $\mu(1)$ consecutive integers from $N$ to $N-\mu(1)+1$. Set a rank counter $r=1$.

Step 5. For each solution $i$ in rank $r$, calculate the distance count $d c_{i}$ using the following equation:

$$
\begin{aligned}
d c\left(P_{i}^{r}\right)=d c_{i} & = \begin{cases}\left\|P_{i}^{r}\right\|_{\infty} / \sigma_{\text {share }}^{r}, & \text { if }\left\|P_{i}^{r}\right\|_{\infty}>\sigma_{\text {share }}^{r} \\
1 \quad & \text { otherwise }\end{cases} \\
\sigma_{\text {share }}^{r} & =\left\|P^{C M(r)}\right\|_{\infty}, P^{C M(r)}=\text { Center of Mass of the set }
\end{aligned}
$$

where $P^{(r)}=\left\{P_{1}^{r}, P_{2}^{r}, \ldots, P_{\mu(r)}^{r}\right\}$

of solutions in rank $r$. The Center of Mass of a group of points is defined as the weighted mean of the points' positions. The weight applied to each point is the point's mass. $\bullet \|_{\infty}$ is the maximum holder metric. Note that $P^{(1)}=P F_{\text {current }}^{\text {restricted }}$.

Calculate the shared fitness using $F_{j}^{\prime}=F_{j} / d c_{j}$.

To preserve the same average fitness, scale the shared fitness as follows:

$$
F_{j}^{\prime} \leftarrow \frac{F_{j} \mu(r)}{\sum_{k=1}^{\mu(r)} F_{k}^{\prime}} F_{j}^{\prime}
$$

Step 6. Increment $\lambda_{j}$ by $\varepsilon^{\prime} \quad\left(\lambda \leftarrow \lambda+\varepsilon^{\prime}\right)$ while $\left[u(\lambda)=u\left(\lambda+\varepsilon^{\prime}\right)\right]$ and $\left[f(\lambda)=f\left(\lambda+\varepsilon^{\prime}\right)\right]$, where $f$ is defined in subsection 5.2.2.

Step 7. If $r<r^{*}$, increment $r$ by one and go to Step 5. Otherwise, the process is complete.

Due to limitations of space, we omit the "Crossover and mutation operators", "Parent selection method", "Population replacement scheme", and "The final step for obtaining a recommendation" subsections.

\section{Computational examples}

\subsection{Test problem 1}

In this section the proposed approach will be applied on a real-life data set. In this data set Leyva and Gastélum (2013) present a real case study dealing with the comparison of municipal socio-demographical situations. It presents the problem situation and an appropriate multicriteria ranking problem formulation. Moreover, a detailed version of the multicriteria evaluation model is presented in the book. The model consists of a complex hierarchy of evaluation models enabling us to take into account the multiple dimensions involved in the evaluation. The set of alternatives to be considered in the evaluation model is represented by such different municipalities of the Sinaloa State, México (Table 1). The problem statement is a relative socio-demographical comparison of such municipali- ties under a ranking purpose, from data of the 2010 Cen-

\begin{tabular}{|c|c|c|c|c|c|}
\hline Label & $\begin{array}{c}\text { Municipalities of } \\
\text { Sinaloa State, } \\
\text { México }\end{array}$ & $\begin{array}{c}\text { Population } \\
2010\end{array}$ & Label & $\begin{array}{c}\text { Municipalities of } \\
\text { Sinaloa State, } \\
\text { México }\end{array}$ & $\begin{array}{c}\text { Population } \\
2010\end{array}$ \\
\hline $\mathrm{a}_{0}$ & Ahome & 416,299 & $a_{9}$ & El Fuerte & 97,536 \\
\hline $\mathrm{a}_{1}$ & Angostura & 44,993 & $a_{10}$ & Guasave & 285,912 \\
\hline $\mathrm{a}_{2}$ & Badiraguato & 29,999 & $a_{11}$ & Mazatlán & 438,434 \\
\hline $\mathrm{a}_{3}$ & Concordia & 28,493 & $\mathrm{a}_{12}$ & Mocorito & 45,847 \\
\hline $\mathrm{a}_{4}$ & Cosalá & 16,697 & $a_{13}$ & Rosario & 49,380 \\
\hline$a_{5}$ & Culiacán & 858,638 & $a_{14}$ & Salvador Alvarade & 79,085 \\
\hline $\mathrm{a}_{6}$ & Choix & 32,998 & $a_{15}$ & San Ignacio & 22,527 \\
\hline$a_{7}$ & Elota & 42,907 & $a_{16}$ & Sinaloa & 88,282 \\
\hline$a_{8}$ & Escuinapa & 54,131 & $a_{17}$ & Navolato & 135,603 \\
\hline \multicolumn{5}{|c|}{ Total of Inhabitants in Sinaloa State ---> } & $2,767,761$ \\
\hline
\end{tabular}
sus of Population and Housing (Data are available on http://www.inegi.org.mx).

Table 1: Municipalities of Sinaloa State, Mexico.

In order to build the valued outranking relation for this data set, we use the ELECTRE-III method. This method requires to define, for each considered criterion, a weight, a preference threshold and a indifference threshold. Since our goal is to illustrate the algorithm, we will not discuss the construction of the valued outranking relation into more detail. Instead, in Table 2, we present the valued outranking relation that we obtained applying the ELECTRE-III method.

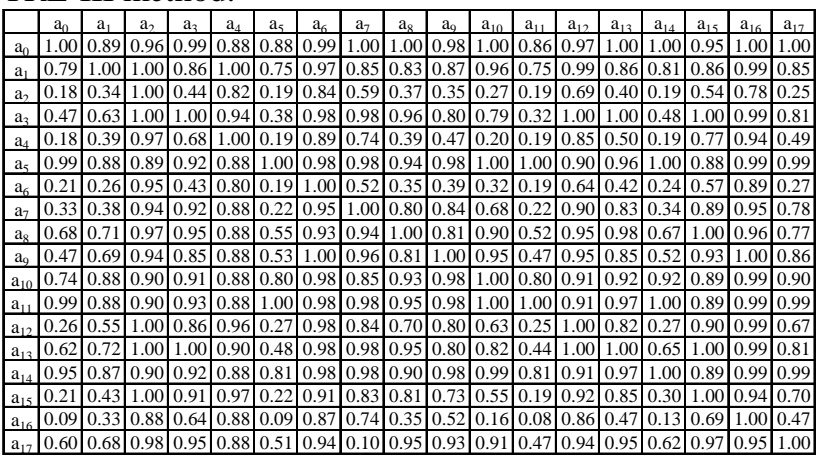

Table 2: Credibility matrix constructed using the ELECTRE-III method.

Table 2 was processed with our proposal of Section 5. The restricted Pareto front found, $P F_{\text {current }}^{\text {restricted }}$, and the associated final set of solutions returned by the MOEA at termination, $P_{\text {current }}^{\text {restrict }}$ are presented in Table 3. 


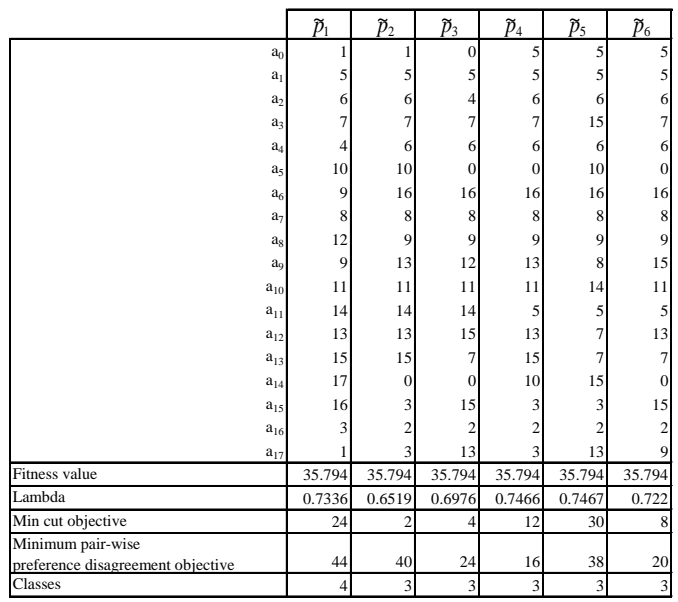

Table 3: Restricted Pareto front found and the associated individuals (genotype) of the solutions space.

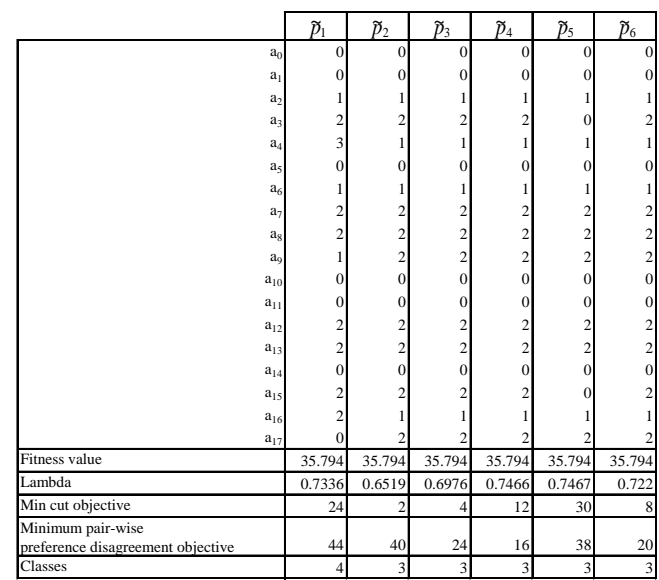

With this information we generate a recommendation for the decision-maker. Tables 3 and 4 suggest a crisp antisymmetric outranking relation, on a set of three classes of alternatives. In Figure 6 we show the results obtained. On the left side we have a table detailing the relations between the alternatives. The alternatives were grouped here by the ranking results found using the presented method. On the right side of the figure we have the representation of the classes of alternatives and the relation between them. In order to simplify the representation, the classes that are above are considered to be preferred to those below. In this case, we have a complete ranking of classes of alternatives. We notice that we have three classes that form a total order. In the table to the left we have highlighted the relations that are in discordance with the ranking result. This result is in concordance with the nature of the data set. Indeed, class 0 corresponds to the municipalities having a less socio-demographic slack. They present a better performance values in the most important decision criteria. Conversely, class 2 includes the municipalities with greater socio-demographic slack. The common characteristic presented by these municipalities is its low performance values in the most important decision criteria; in the middle, the class 1 are the municipalities which vary its performance values in the decision criteria. This leads us to conclude that we have obtained a natural relational structure underlying the data set. Hence, from our point of view, this example gives a good motivation for our ranking method, since it confirms relations between classes that could have been brought out a priori by other means.

Table 4. Restricted Pareto front found and the associated individuals (fenotype) of the solutions space.
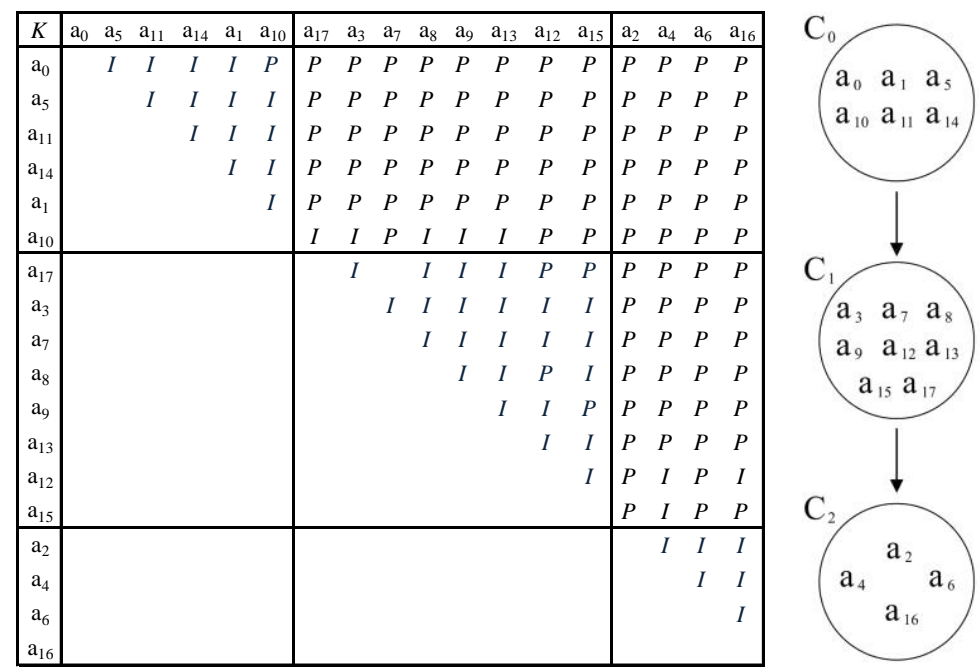

Fig. 6. Ranking result on Municipal Socio-Demographic Comparison problem in Sinaloa State, Mexico. 


\section{Conclusions and future research}

In this paper, we have addressed the problem of multicriteria ranking with a medium-sized set of alternatives. The main added value of this contribution is to propose a multiobjective evolutionary approach that can be applied on a valued outranking relation to solve this problem. A distinctive feature of the approach relies on the fact that it takes the multiobjective nature of the multicriteria ranking method into account. It helps the decision maker to identify both a partition of the set of alternatives in classes and preference relations between the classes. In this sense, it differs from classical approaches of multicriteria ranking. A validation test has been conducted on a real data set. We have been able to show that the resulting complete ranking of classes of alternatives is robust and meaningful. From our point of view, the elicitation of crisp antisymetric outranking relation between the classes offers additional information to the decision maker that can help him have a better insight into the multicriteria structure of the data set. We will use in the future an empirical approach to test our method on a medium set of benchmarks with a wide variety in their structure, for highlighting the efficiency of the proposed method. Validation tests will be conducted on both artificial and real data sets. We would like to show how close the results from our method come to the optimal solutions. It will also be important to explore the limits of this approach, by finding the top size within instances can be solved with acceptable performance. We will address these issues in the future.

\section{References}

[1] Bui, T. Nguyen, and Curt Jones. "Finding good approximate vertex and edge partitions is NP-hard", Information Processing Letters, 42-3(1992): 153-159.

[2] Bui, T. Nguyen, and Byung B. Moon. "Genetic algorithm and graph partitioning", IEEE Transactions on Computers, 45-7(1996): 841-855.

[3] Coello, Carlos A., David A. Van Veldhuizen, and Gary B. Lamont. Evolutionary Algorithms for Solving Multi-Objective Problems. New York: Kluwer Academic Publishers, 2002.

[4] Cook, W.D. and L.M. Seiford. "Priority Ranking and Consensus Formation". Management Science, 24-16 (1978): 1721-1732.

[5] Cormen, Thomas H., Charles E. Leiserson, Ronald L. Rivest, and Clifford Stein. Introduction to Algorithms. Cambridge, MA: MIT Press, 2001.

[6] De Smet, Yves, and Stefan Eppe. "Relational multicriteria clustering: The case of binary outranking matrices", In Proceedings of the 5th International Conference on Evolutionary Multicriterion Optimization. Volume 5467 of Lecture Notes in Computer Science. Edited by Matthias Ehrgott pp. 380-392. Berlin. Springer, 2009.
[7] Doumpos, Michael, and Constantin Zopounidis. Multicriteria Decision Aid Classification Methods. Dordrecht: Kluwer Academic publishers, 2002.

[8] Ehrgott, Matthias, José R. Figueira, and Salvatore Greco. Trends in Multiple Criteria Decision Analysis. New York, U.S.A.: Springer Science + Business Media, Inc, 2010.

[9] Figueira, José, Salvatore Greco, and Matthias Ehrgott. Multiple Criteria Decision Analysis: State of the Art Surveys. New York: Springer Science + Business Media, Inc., 2005.

[10] Fodor, János, and Marc Roubens. Fuzzy Preference Modeling and Multicriteria Decision Support. Dordrecht: Kluwer Academic Publishers, 1994.

[11] French, Simon. Decision Theory: An Introduction to the Mathematics of Rationality. New YorkChicester-Brisbane-Toronto: Halsted Press, 1986.

[12] Fonseca, C.M. and P.J. Fleming. "Genetic Algorithms for Multiobjective Optimization: Formulation, Discussion and Generalization". In Proceedings of the Fifth International Conference, pp. 416-423, San Mateo, CA: Morgan Kaufmann, 1993.

[13] Guitouni, Adel, and Jean-Marc Martel. "Tentative guidelines to help choosing an appropriate MCDA method", European Journal of Operational Research, 109 (1998): 501-521.

[14] Keeney Ralph L., and Howard Raiffa. Decision with Multiple Objectives: Preferences and Value Tradeoffs. New York: Wiley, 1976.

[15] Kemeny, John G., and James L. Snell. "Preference Ranking: An Axiomatic Approach". In Mathematical Models in the Social Sciences. Boston: Ginn, 1962.

[16] Lee, Y., McKay, R., Nguyen, X. and Kim, D., "The effect of vesicular selection in dynamic environments", Proceedings of the 11th Annual conference on Genetic and evolutionary computation, pp. 1867-1868, ACM, 2009.

[17] Leyva, Juan C., and Mario Araoz M. "A multiobjective extension of the net flow rule for exploiting a valued outranking relation", Int. J. Multicriteria Decision Making 3 (2013): 36-54.

[18] Leyva, Juan C., and Diego A. Gastélum. Evaluación del Desarrollo Socioéconomico de Sinaloa bajo un Enfoque Multicriterio. México: Juan Pablos Editor, 2013.

[19] Park, Y.J. and M.S. Song. "A genetic algorithm for clustering problems", In Proceedings of the Third Annual Conference on Genetic Programming, pp. 568-575, Madison, WI: Morgan Kaufmann, 1998.

[20] Roy, Bernard. Multicriteria Methodology for Decision Aiding. Dordrecht: Kluwer Academic Publishers, 1996.

[21] Roy, Bernard, "The Outranking Approach and the Foundations of ELECTRE Methods". In Reading in Multiple Criteria Decision Aid. Edited by Bana e Costa, C.A. pp. 155-183. Berlin: Springer-Verlag, 1990. 
[22] Syswerda, Gilbert. "Uniform Crossover in Genetic Algorithms". In Proceedings in the Third International Conference on Genetic Algorithms. 2-9. San Mateo, CA: Morgan Kauffman Publishers, 1989.

[23] Triantaphyllou, Evangelos. Multicriteria Decision Making Methods: A Comparative Study. Boston MA: Kluwer Academic Publishers, 2000.

[24] Vincke, Philippe, Multicriteria Decision-aid. Chichester: Wiley, 1992.

[25] Xiaozhan, Xu, Jean-Marc Martel, and Bernard F. Lamond. "A multiple criteria ranking procedure based on distance between partial preorders", European Journal of Operational Research, 133-1 (2001): 6980. 